\title{
Worldlines in the Einstein's Elevator
}

\author{
Mathieu Rouaud \\ Boudiguen 29310 Querrien, FRANCE, mathieu137@gmail.com
}

\begin{abstract}
We all have in mind Einstein's famous thought experiment in the elevator where we observe the free fall of a body and then the trajectory of a light ray. Simply here, in addition to the qualitative aspect, we carry out the exact calculation. We consider a uniformly accelerated reference frame in rectilinear translation and we show that the trajectories of the particles are ellipses centered on the horizon of the events. The frame of reference is non-inertial, the spacetime is flat, the metric is non-Minkowskian and the computations are performed within the framework of special relativity. The deviation, compared to the classical case, is important close to the horizon, but small in the box, and the interest is above all theoretical and pedagogical. The study helps the student to become familiar with the concepts of metric, coordinate velocity, horizon, and, to do the analogy with the black hole.
\end{abstract}

Keywords: special relativity, Einstein, elevator, lift, horizon, accelerated, ellipse, circle.

\section{INTRODUCTION}

We imagine a portion of empty space infinitely distant from all masses. We have a large box in which an observer floats in weightlessness. With the help of a hook and a rope, a constant force is exerted on the box thus animated by a rectilinear translation motion uniformly accelerated. The observer then experiences an artificial gravity. We will study in the elevator's frame the motion of light, then of a massive particle, and, finally, we will do a comparison with the black hole during a free fall from rest.

In the frame of the box, first inertial, a light beam propagates at speed $c$ along a straight-line trajectory. Then, the box is accelerated and a ray, initially perpendicular to the direction of motion, follows a curved trajectory. Let us quote Albert Einstein in his book Relativity ${ }^{[1]}$ : «It can easily be shown that the path of the same ray of light is no longer a straight line ».

Following the birth of special relativity in 1905, the accelerated elevator thought experiment, proposed by Einstein as early as 1908, allowed to draw the analogy with gravity to develop an intuition and to guide the foundation of general relativity. New theory of gravitation established in 1915 which then supplanted Newton's theory. This image was used to illustrate the principle of equivalence and to predict the deviation of light rays by a massive star.

Nevertheless, the historical development of the theory should not hide an essential point: the deviation of a light ray in the accelerated elevator is fully explained within the framework of special relativity. Indeed, in the accelerated elevator frame of reference, spacetime remains flat, no gravitational field here, and the deviation of light rays is understood by a purely kinematic reasoning.

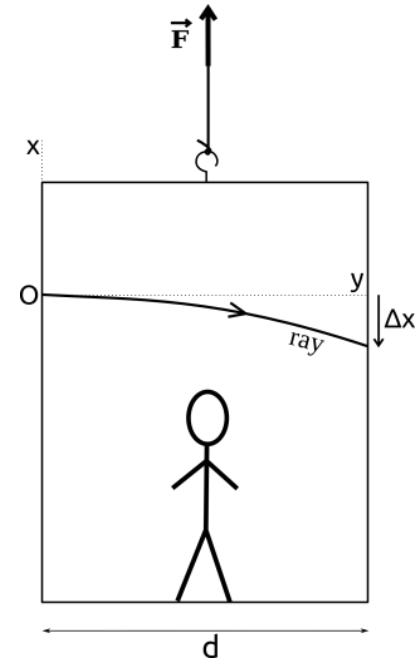

Fig. 1 The Einstein's elevator.

According to Einstein's second postulate, the speed of light in vacuum is constant and equal to $c$ in all inertial frames of reference. A logical consequence of this postulate: in a non-inertial frame the speed of light can, a priori, be different than $c^{1}$. Another property: a free particle follows a rectilinear and uniform trajectory in an inertial frame. Therefore, a free particle can follow a curved trajectory in a non-inertial frame. As we will show, this is precisely what happens here for the ray of light.

1 It remains, of course, that an object can in no case exceed the speed of light, and that the speed of light for a local Minkowskian observer is always equal to $c$. 


\section{UNIFORMLY ACCELERATED REFERENCE FRAME}

\subsection{Coordinate system}

We want to describe physics from the point of view of the accelerated observer. A reference frame is a physical entity to which we associate a coordinate system for localization. A uniformly accelerated reference frame $R$ is defined as a set of observers who remain at rest with respect to each other in a hypothetical rigid three-dimensionnal structure. We imagine a continuous set of infinitesimal observers each equipped with a ruler and a clock ${ }^{[2,3]}$. An event is uniquely specified with the observer on whose worldline the event occurs. We decide to assign a set of three numbers $(x, y, z)$ to each observer and a number $t$ at each point on its worldline. For an inertial frame all the clocks can be synchronized and $t$ corresponds to the proper time of each observer at rest in $R^{\prime}$. For a non-inertial frame, this is no longer possible and each observer has a second clock called coordinate clock. However, the uniformly accelerated frame is still rigid, because the relationships between the observers remain unchanged. We choose an observer $O$ of $R$ used as reference: $x=0$ and in his particular case the proper and coordinate times remain equal. We assign an $x$ to each observer by shifting the ruler as much as necessary in the direction of the proper acceleration given by an accelerometer. We proceed in the same way in two orthogonal directions for $y$ and $z$. O emits a periodic light signal and each of the other observers $\mathrm{X}$ equals the rate of its coordinate clock with the signal received from $\mathrm{O}$. To synchronize all the coordinate clocks we use the radar method: $\mathrm{O}$ sends a signal at $t_{1}$ reflected by $\mathrm{X}$ and back to $\mathrm{O}$ at $t_{2}$. When the signal is reflected by $\mathrm{X}$ the time $t=\left(t_{1}+t_{2}\right) / 2$ is assigned to its coordinate clock. A coordinate clock can be compared to a clock radio-controlled by O's proper clock.

The coordinate system has been built intrinsically with the non-inertial observers. To define a constant acceleration for $\mathrm{O}$ in an inertial frame $R^{\prime}$, we consider $R^{\prime}$ coinciding instantaneously with $R$ and then it is easy to show that the proper acceleration $a_{p}$ is equal to the acceleration $a$ of $R^{\prime}$ with respect to $R$. At this instant all the clocks are set to zero. If later at $t$, a second inertial frame $R^{\prime \prime}$ coincides with $R$, the set of coordinate clocks of $R$ will still be synchronized and we will be able to equalize their dates with the set of proper clocks of $R^{\prime \prime}$, however the set of proper clocks of $R$ will no longer be synchronized especially as $x$ is large.

When a particle passes close to an observer, to measure velocity and acceleration, he can use one or the other of his clocks: coordinate velocity and acceleration, $v^{\mu}=d x^{\mu} / d t$ and $a^{\mu}=d v^{\mu} / d t$, or local, $d x^{\mu} / d \tau$ and $d^{2} x^{\mu} / d \tau^{2}$. In an inertial reference frame the speed of light is constant and we can indifferently measure distances with a ruler or a radar. In a non-inertial frame the coordinate velocity of the light varies and the distances measured by each method are generally different ${ }^{[4]}$.

\subsection{Metric and change of coordinates}

In a non-inertial frame of reference the metric is non-Minkowskian. We consider the frame $R$ in rectilinear translation and uniformly accelerated with respect to $R^{\prime}$ inertial. We have the coordinate system (ct, $x, y, z)$ and we give the metric for a constant proper acceleration $a$ along the $x$-axis ${ }^{[5,6]}$ :

$$
\text { For a particle: } d s^{2}=c^{2} d \tau^{2}=g_{\mu \nu} d x^{\mu} d x^{v}=\left(1+\frac{a x}{c^{2}}\right)^{2} c^{2} d t^{2}-d x^{2}-d y^{2}-d z^{2} \text { with } \vec{a}=a \vec{u}_{x}
$$

The calculation shows that all the components of the Riemann curvature tensor are zero ${ }^{[7]}$, so the spacetime is flat, and there is a global change of coordinates from $R$ to $R^{\prime[5]}$ :

$$
\begin{gathered}
c t^{\prime}=\left(x+\frac{c^{2}}{a}\right) \operatorname{sh}\left(\frac{a t}{c}\right), \quad x^{\prime}=\left(x+\frac{c^{2}}{a}\right) \operatorname{ch}\left(\frac{a t}{c}\right)-\frac{c^{2}}{a}, \quad y^{\prime}=y, \quad z^{\prime}=z, \\
\text { with } \quad \vec{u}_{x^{\prime}}=\vec{u}_{x}, \quad x^{\prime}\left(t^{\prime}=0\right)=x(t=0)=0 \quad \text { and } \quad v_{x^{\prime}}^{\prime}\left(t^{\prime}=0\right)=0 .
\end{gathered}
$$

We find the Minkowski metric in $R^{\prime}: d s^{2}=d s^{\prime 2}=c^{2} d t^{\prime 2}-d x^{\prime 2}-d y^{\prime 2}-d z^{\prime 2}$.

To simplify the study, we use dimensionless quantities. For the distances, $\mathrm{X}=\mathrm{x} / \mathrm{d}_{\mathrm{H}}$ with the horizon distance $\mathrm{d}_{\mathrm{H}}=\mathrm{c}^{2} / \mathrm{a}$. For times, $\mathrm{T}=\mathrm{t} / \mathrm{t}_{\mathrm{H}}$ with the horizon time $\mathrm{t}_{\mathrm{H}}=\mathrm{c} / \mathrm{a}$. For an acceleration equivalent to the intensity of gravity at the earth's surface, the horizon quantities are approximately one light-year and one year. To further illustrate our point, we can associate the reference frame $R^{\prime}$ to the galactic reference frame where the stars are supposed to be fixed, and replace the elevator frame $R$ by the rocket frame which makes an interstellar voyage. The hyperplane $\mathrm{x}=-\mathrm{d}_{\mathrm{H}}$ defines the event horizon as a break in the causal link. The astronauts will see the Earth, their starting point, moving away then stopping at a light-year with a proper time frozen at one year. 
Fig. 2 Minkowski diagram in $R^{\prime}$ inertial. The uniformly accelerated rocket has a hyperbolic worldline. We have represented the coordinate lines of $R^{\prime}$ and $R$ in $R^{\prime}$. In both cases, the coordinate lines of space and time are orthogonal. In this case, the rocket defines a rigid body of reference ${ }^{[2,5,3]}$ with respect to which we can study the trajectory of the light rays.

The proper acceleration felt by an observer varies with $x: a_{p}=a /(1+X)$.

Fig. 3 The worldlines of 12 particular rays in a Minkowski diagram $\left(T^{\prime}, X^{\prime}, Y^{\prime}\right)$ with $\theta^{\prime}\left(t^{\prime}=0\right)=\theta(t=0)$ as initial conditions.
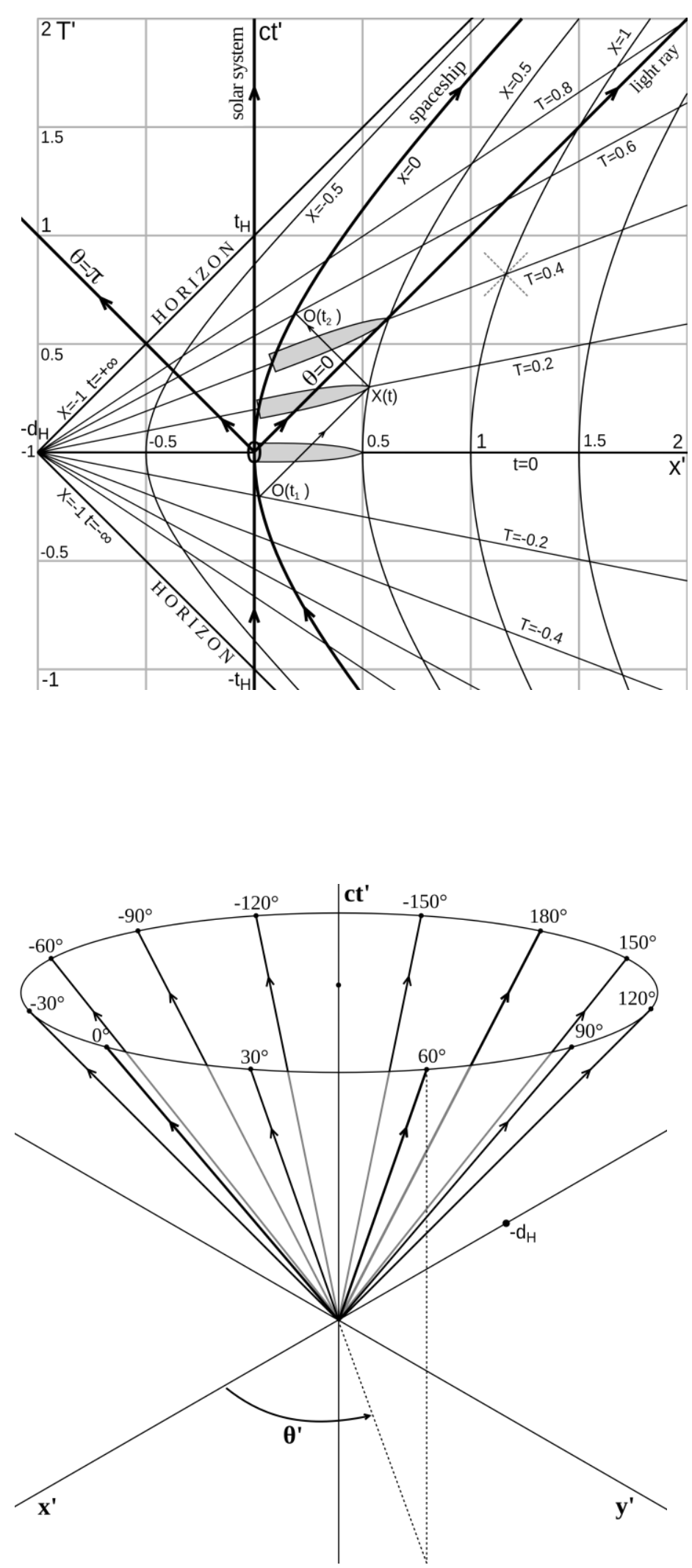


\section{RAYS OF LIGHT}

Without losing in generality, we take as initial condition a light ray that enters the elevator when it has a zero speed in the inertial reference frame. All clocks are then set to zero, and the angle of entry of the ray is the same for all observers. The trajectories are all located in the plane $z^{\prime}=z=0$.

\subsection{Lateral rays}

In $R^{\prime}$, the ray worldline equations are $\mathrm{Y}^{\prime}=\mathrm{T}^{\prime}$ and $\mathrm{X}^{\prime}=0$. Also $\mathrm{Y}^{\prime}=\mathrm{Y}$, hence we have ${ }^{[7]}$ :

$$
X=1 / \operatorname{ch} T-1, \quad Y=\operatorname{th} T \quad \text { and } \quad(X+1)^{2}+Y^{2}=1
$$

The ray of light traces a quarter circle of radius $d_{H}$ and center $\left(-d_{H}, 0\right)$. For a light ray $d \tau=0$ then $|\beta|=$ $1+X$ with $\beta=d L / d T .|\beta| \neq 1$ because the coordinate system $(x, t)$ uses different physical methods of measuring distance and time: rods for $x$ and O's clock for $t$. The coordinate velocity of the light ray, initially equal to $c$, decreases and becomes zero on the horizon in an infinite time. As the components of the metric tensor depend on the point, the coordinate velocity corresponds to the proper velocity just for the particular observer $\mathrm{O}$, whereas for an inertial frame this velocity keeps the same meaning for all observers. For example, for the uniformly accelerated frame, the notion of rigidity is maintained, but, on the other hand, we can no longer define a set of proper clocks synchronized on the worldline of a particle. For the observer $O$, the measurements of his observers placed at different $x$ 's with their coordinate clock tell him that everything goes slower downwards, and faster upwards. This is why from his point of view light goes slower at the lower levels, while a lower observer measures, where he is with is proper clock, a speed of light well equal to $c$.

For comparison, we give the predictions of Newton's theory: $X=-Y^{2} / 2$ and $\beta=\sqrt{1-2 X}$. In this approximation the trajectory is parabolic and the speed of light increases towards infinity.

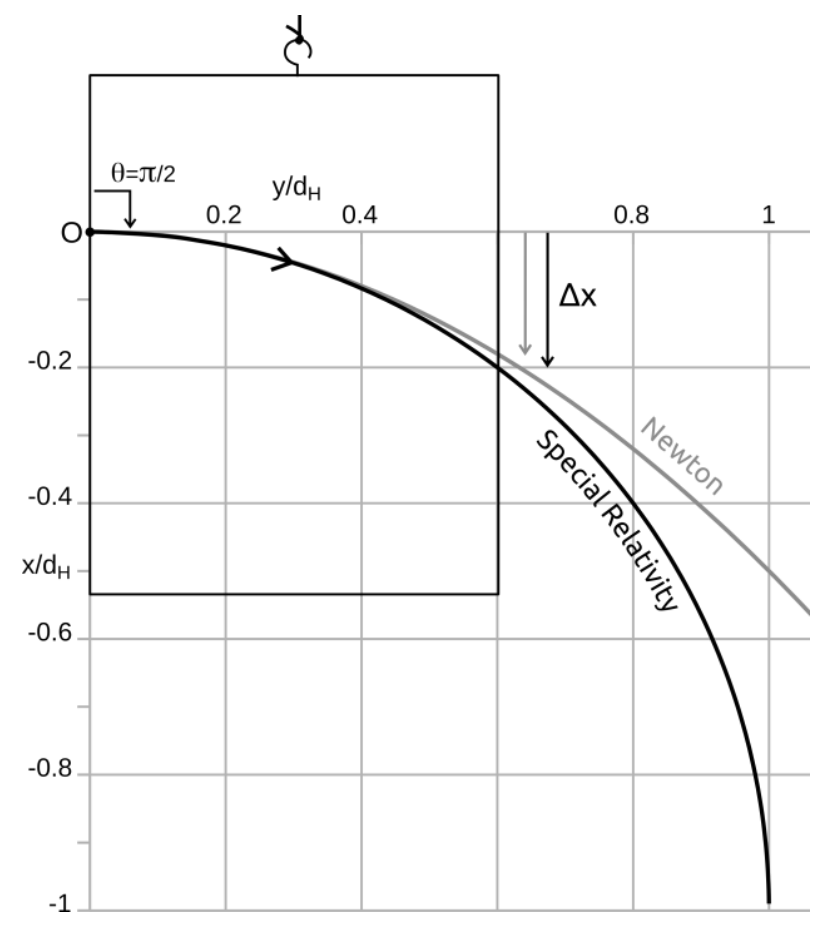

Fig. 5 On the right, the speed of light which decreases and tends towards zero in $x=-d_{H}$. In classical theory, there is no horizon and the speed of light increases.
Fig. 4 On the left, the circular trajectory of the ray traced on a vertical wall of the elevator. The deviation is greater than that of Newton's parabolic trajectory. At the level of the horizon the deviation is double.

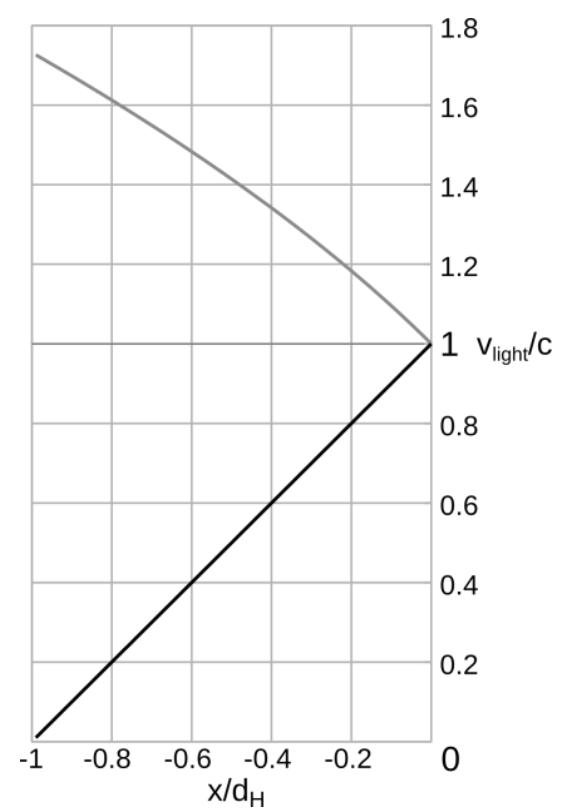

\subsection{Any rays}

In general, the worldline of a ray in $R^{\prime}$ has for equations $Y^{\prime}=T^{\prime} \sin \theta^{\prime}$ and $X^{\prime}=T^{\prime} \cos \theta^{\prime}$. The calculation gives $(X+1)^{2}+(Y-1 / \tan \theta)^{2}=1 / \sin ^{2} \theta$. The ray of light describes a circular trajectory of radius $d_{H} /|\sin \theta|$ and center $\left(-d_{H}, d_{H} / \tan \theta\right)$. The speed of a luminous ray, whatever the initial conditions of the trajectory, varies linearly with $\mathrm{X}$. If $\mathrm{X}>0$ the ray goes faster than $c$, and if $\mathrm{X}<0$ the speed of light becomes less than $\mathrm{c}$ and tends towards zero when $\mathrm{X}$ tends towards -1 . 
Newton's parabolic trajectory: $X=-Y^{2} /\left(2 \sin ^{2} \theta\right)+Y / \tan \theta$. For example, if the light ray initially goes forward, its speed first decreases, then the ray returns downward and its speed increases and tends towards infinity.

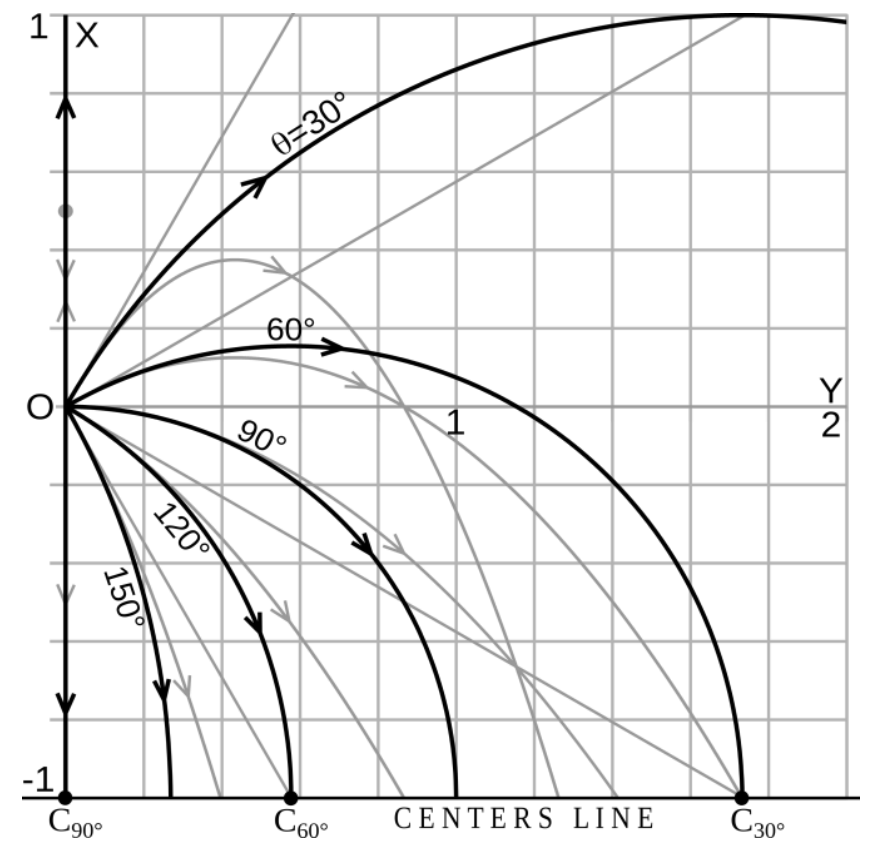

Fig. 6 In the $(X, Y)$ plane, the trajectories of nonvertical light rays are portions of circles centered on the horizon. The rays thus arrive perpendicularly to the line of centers which is identified with the horizon. The grayed straight lines correspond to the inertial trajectories, in case the rocket is not accelerated. The gray parabolic lines are the Newtonian trajectories.

Fig. 7 Below is the Minkowski diagram in the noninertial reference frame $(T, X, Y)$. Apart from the limit case $\theta=0$, all the light rays follow a partially helical worldline that asymptotically joins the horizon plane $x=-d_{H}$ in an infinite time $t$. The maximum deviation tends towards the half-turn for a ray emitted in the direction close to $\theta=0^{\circ}$.

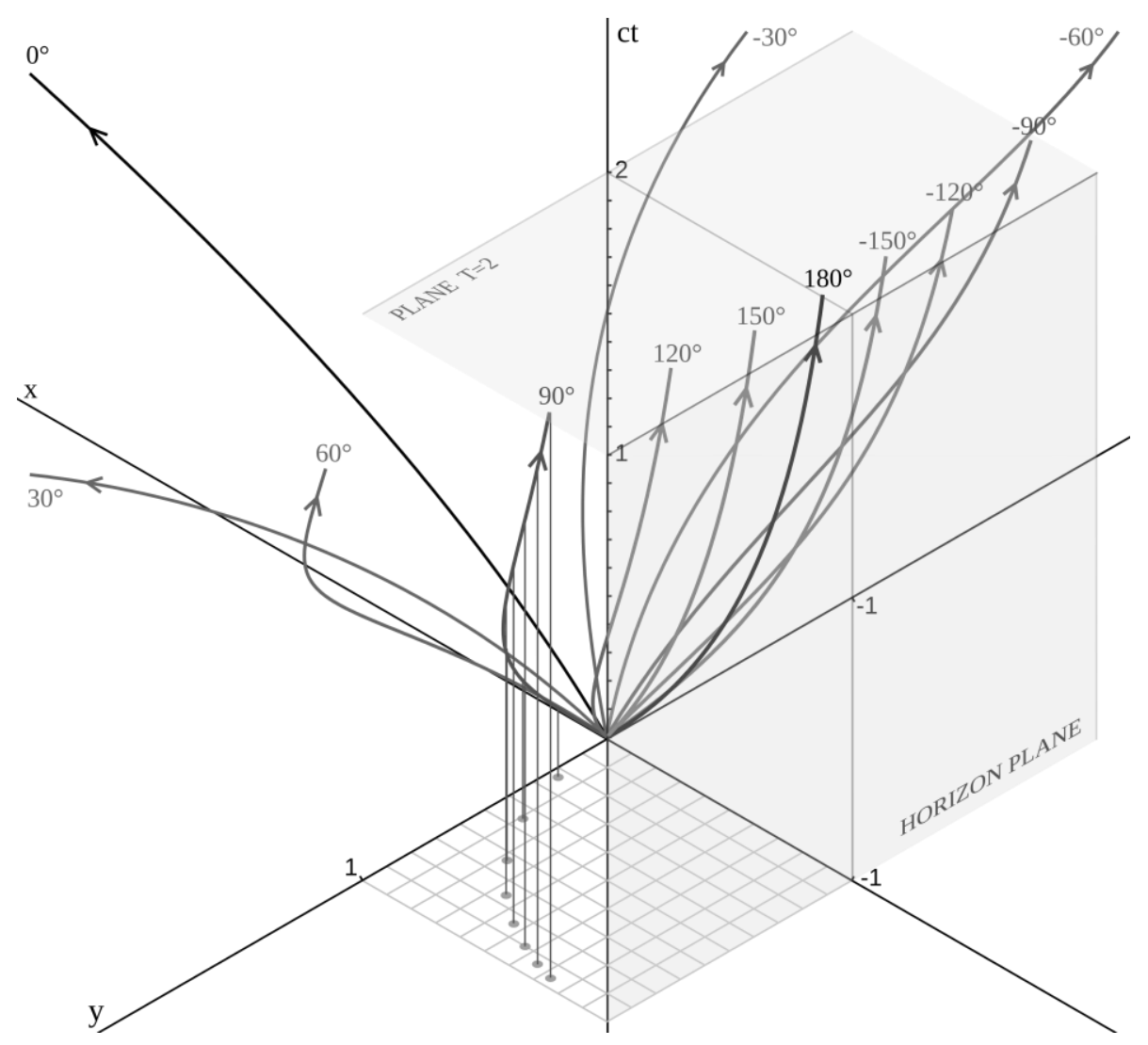

For vertical rays $\beta=d X / d T= \pm(1+X)$ and worldlines have for equations $Y=0$ and $T= \pm \ln (1+X)$. A notable difference from the classical theory is that the relativistic rocket cannot overtake the ray sent forward. 


\section{NON-ZERO MASS PARTICLES}

\subsection{Trajectories and worldlines}

The worldline of a particle in $R^{\prime}$ has for equations $Y^{\prime}=\beta_{0} T^{\prime} \sin \theta^{\prime}$ and $X^{\prime}=\beta_{0} T^{\prime} \cos \theta^{\prime}$ with $\beta_{0} C$ the initial velocity. The calculation gives $(X+1)^{2} / R_{X}{ }^{2}+\left(Y-Y_{C}\right)^{2} / R_{Y}{ }^{2}=1$ with $R_{X}=a / d_{H}=$ $1 / \sqrt{1-\beta_{0}^{2} \cos ^{2} \theta}$ and $R_{Y}=b / d_{H}=\beta_{0}|\sin \theta| R_{X}{ }^{2}$. The particle describes an elliptical trajectory of semimajor axis $a$, of semi-minor axis $b$ and center $\left(-d_{H}, \beta_{0}^{2} \sin \theta \cos \theta R_{X}^{2} d_{H}\right)$.

Newton's parabolic trajectory: $X=-Y^{2} /\left(2 \beta_{0}^{2} \sin ^{2} \theta\right)+Y / \tan \theta$.

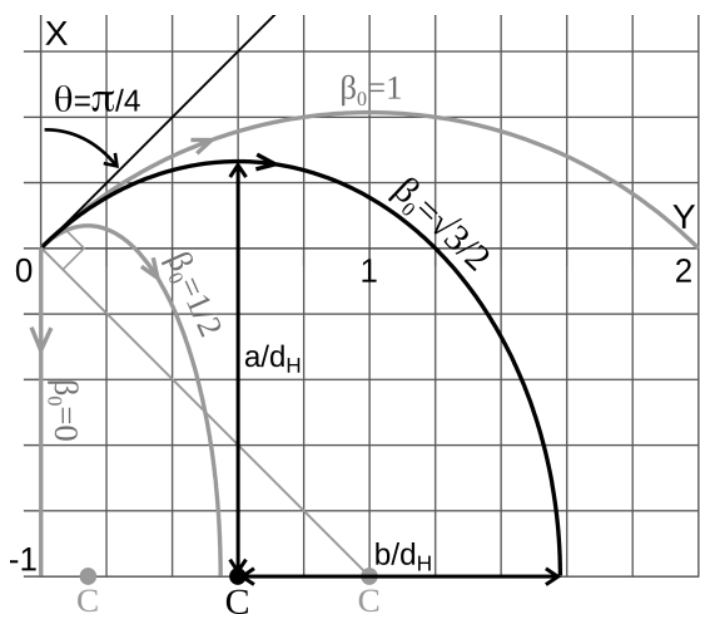

Fig. 8 On the left, in the $(X, Y)$ plane, the trajectories of the particles are portions of ellipses centered on the horizon. The trajectories are perpendicular to the horizon line.

Fig. 9 On the right, the Minkowski diagram in the non-inertial reference frame $(T, X, Y)$. Worldlines for $v_{0}=50 \%$ of $c$. In bold the worldline for a particle released at rest.

Worldlines equations:

$$
\left\{\begin{array}{c}
X=\frac{1}{\operatorname{ch} T-\beta_{0} s h T \cos \theta}-1 \\
Y=\frac{\beta_{0} \operatorname{shT} \sin \theta}{\operatorname{ch} T-\beta_{0} \operatorname{shT} \cos \theta}
\end{array}\right.
$$

The intersection between a horizontal plane and the tubular worldsheet, formed by the set of worldlines for different $\theta$ and a given $\beta_{0}$, also forms an ellipse (dotted curve).

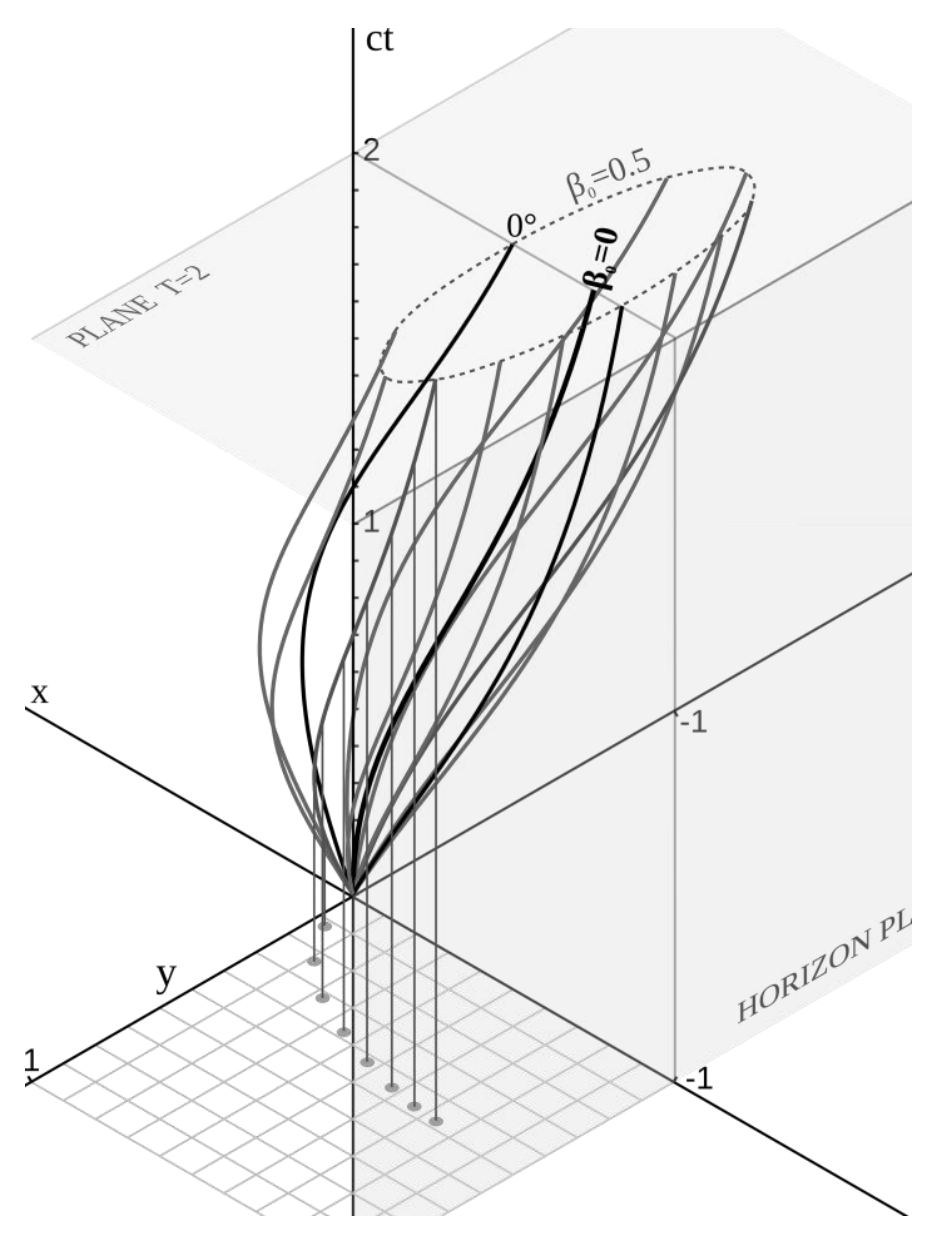

Although the underlying theory is known for more than a century, to my knowledge, the specific results obtained here, an elliptical trajectory for a massive particle, and previously a circular trajectory for a ray of light, are new.

\subsection{Velocity and acceleration for a particle released at rest}

The initial speed of the particle is zero and the motion is vertical:

$$
\left\{\begin{array}{c}
X=\frac{1}{c h T}-1 \\
\beta=\frac{d X}{d T}=-\frac{t h T}{c h T}=-\sqrt{1-(X+1)^{2}}(X+1) \\
A=\frac{d \beta}{d T}=\frac{c h^{2} T-2}{c h^{3} T}=\left[1-2(X+1)^{2}\right](X+1)
\end{array}\right.
$$


We have: $\quad v_{\max }=\frac{c}{2} \quad$ at $\quad X\left(v_{\max }\right)=\frac{1}{\sqrt{2}}-1 \simeq-0.3^{[8,3]}$

Initially the speed increases, then it decreases and tends to zero on the horizon. The acceleration is first negative, then zero and becomes positive. The elevator observer can initially interpret the metric effects as a inertial force similar to gravitation, but then his measurements will lead him to interpret a repulsive force as the particle approaches the horizon. These are spatiotemporal perspective effects experienced by the observer of the rocket. These metric effects due to the non-inertial character of the frame of reference are sometimes described in terms of fictitious forces, because the particle in $R^{\prime}$ inertial does not undergo any force and follows a rectilinear and uniform trajectory.

For Minkowskian observers, instantaneously at rest in successive inertial reference frames that coincide at every moment with $R$ :

$$
\begin{array}{r}
d t_{\text {Mink }}=(1+X) d t \text { then } v_{\text {Mink }}\left(x_{v_{\text {max }}}\right)=c / \sqrt{2} \simeq 71 \% c \\
\text { also } v_{\text {max }}=v_{\text {lum }} / \sqrt{2}
\end{array}
$$

For the proper time, the metric gives $\tau(X)=t_{H} \sqrt{1-(1+X)^{2}}$. In the plane $\left(\tau / t_{\mathrm{H}}, X\right)$ we have a quarter circle. For example, when the falling speed is maximum, $T=\operatorname{argch} \sqrt{2} \simeq 0,9$ and $\tau / t_{H}=1 / \sqrt{2} \simeq 0,7$. And for the observer of the vessel at $O$, the time of the object in free fall freezes, and tends towards $t_{\mathrm{H}}$ when the time of the clock at $O$ tends towards infinity. When a free-falling observer crosses the horizon, apart from the breaking of the causal link with the vessel, nothing special happens and his proper time continues to elapse indefinitely.

Fig. 10 Top right, the Minkowski diagram in the non-inertial frame $(T, X)$. The cones indicate how the coordinate velocity of light varies with respect to $c$.

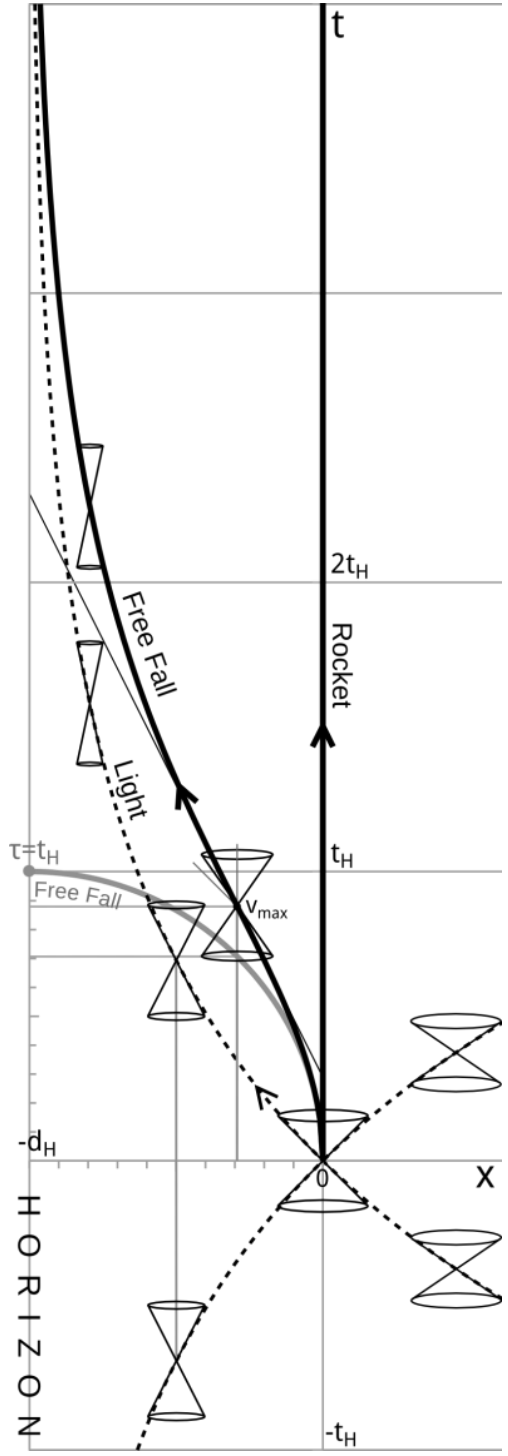

Fig. 11 Falling velocity of a particle released at rest from Fig. 1 Particle acceleration during a free fall in the $\mathrm{O}$ at $T=0$. In gray the classical case.

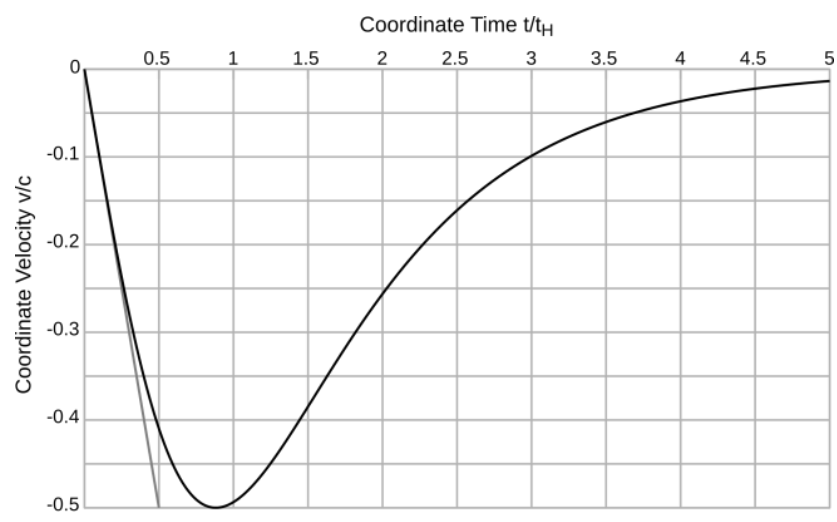
uniformly accelerating reference frame.

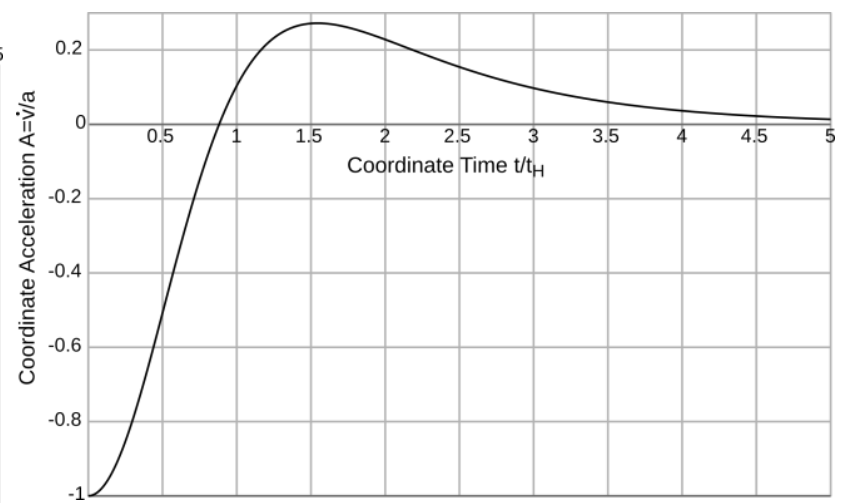

\subsection{Comparison with the black hole}

To broaden our view we will look at another non-inertial frame of reference $R$ defined by the Schwarzschild metric. We have an observer at far distance from a spherical, static, neutral and of mass $M$ star. There is also a horizon and the velocity and acceleration curves show analogies. On the other hand space-time is curved, there is no global change of coordinates to an underlying inertial reference frame, tidal effects will be present, free fall is no longer adiabatic because of the emission of gravitational waves, and the metric changes during the fall if the body is of non-negligible mass in front of the main star ${ }^{[9,10]}$. We will use the 
Lagrangian approach to determine the equation of motion. The free-falling particle maximizes its proper time and follows a geodesic. We limit the study to the adiabatic radial fall of a test mass with an velocity of zero to infinity. The differential equation is then solved by a numerical approach for the plotting of the curves.

For a particle: $d s^{2}=c^{2} d \tau^{2}=g_{\mu \nu} d x^{\mu} d x^{\nu}=g(r) c^{2} d t^{2}-\frac{d r^{2}}{g(r)}$

$$
\text { with } g=1-\frac{r_{S}}{r} \text { and } r_{S}=\frac{2 G M}{c^{2}}
$$

Lagrangian: $\tau=\int L(r, v) d t, \quad L=\sqrt{g-\frac{\beta^{2}}{g}}$ and $L-\frac{\partial L}{\partial v} v=c s t$

We obtain: $\beta=-\left(1-\frac{1}{R}\right) \frac{1}{\sqrt{R}}$ and $A=\frac{d \beta}{d T}=\frac{\ddot{r}}{a_{S}}=\frac{1}{2 R^{2}}\left(1-\frac{1}{R}\right)\left(\frac{3}{R}-1\right)$

$$
\text { with } R=\frac{r}{r_{S}}, \quad T=\frac{t}{t_{S}}, \quad t_{S}=\frac{r_{S}}{c} \text { and } a_{S}=\frac{c^{2}}{r_{S}}
$$

We have $v_{\text {max }}=2 /(3 \sqrt{3}) c \simeq 38 \% c$ at $r\left(v_{\max }\right)=3 r_{S}{ }^{[11]}$. Here too the speed reaches a maximum and the acceleration changes of sign. The metric effects correspond to gravitation, they cannot be canceled over the whole space by a change of coordinates and they are well real.

For Minkowskian observers: $d t_{\text {Mink }}=\sqrt{g} d t$ and $d r_{\text {Mink }}=d r / \sqrt{g}$ then $v_{\text {Mink }}\left(r_{v_{\text {max }}}\right)=c / \sqrt{3} \simeq 58 \% c$ and $v_{\text {max }}=v_{\text {lum }} / \sqrt{3}$

$$
\text { with } v_{\text {lum }}\left(r_{v_{\max }}\right)=2 / 3 c \text {. }
$$

For the proper time the metric gives $\tau(R)=2 / 3\left(R_{0}{ }^{3 / 2}-R^{3 / 2}\right) t_{S}$. For example, with the initial condition $\tau=0$ when $\mathrm{T}=0$ at $\mathrm{R}_{0}=10$, when the speed of fall is maximum, $T \simeq 21$ and $\tau / t_{S} \simeq 18$. For an observer outside the black hole, the time of the falling object freezes on the horizon when the time of its clock tends towards infinity. Whereas for the observer in free fall, a finite proper time of about 2.8 ts elapses between the maximum speed and the crossing of the horizon, and, apart from the breaking of the causal link with the outside, nothing special happens during the crossing. Then $2 / 3$ ts of proper time is added until the singularity where the observer is destroyed.

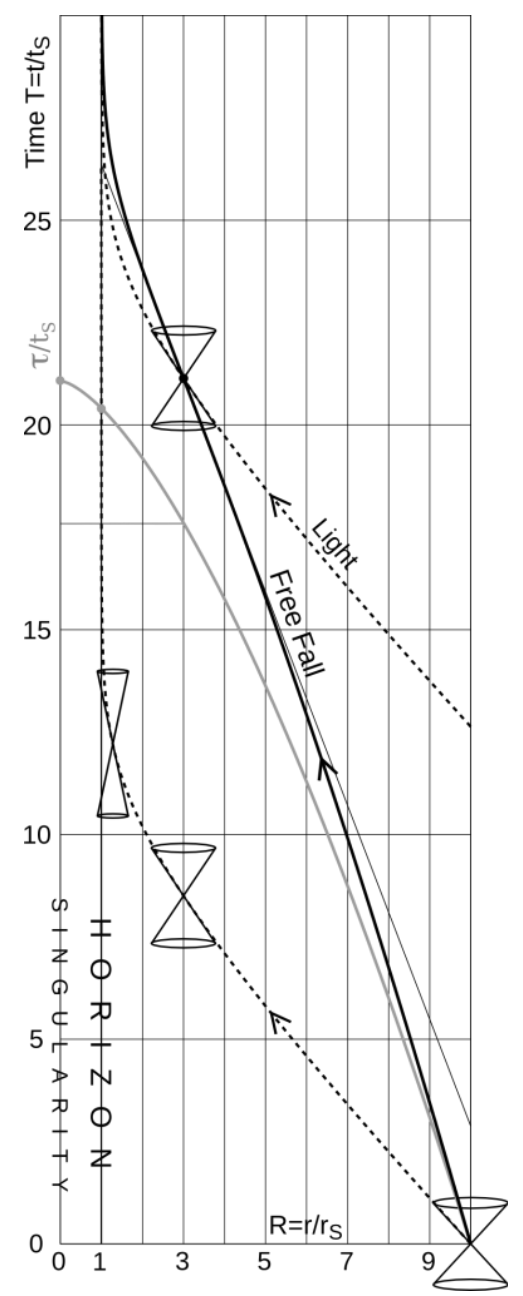

Fig. 13 Minkowski diagram in the Schwarzschild frame $(T, R)^{[12,13]}$.

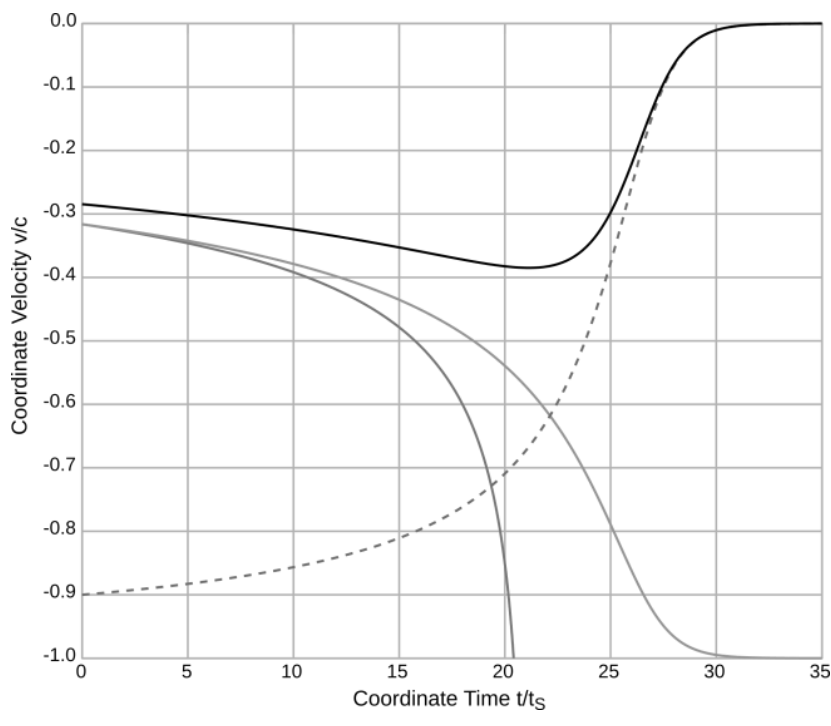

Fig. 15 On the right, the acceleration of the particle in radial fall towards a black hole. In gray the classical curve.
Fig. 14 On the left, falling velocity of a particle dropped without velocity from infinity. In dark gray the curve according to Newton's laws. Dotted the speed of light. In light gray, the speed for a local observer.

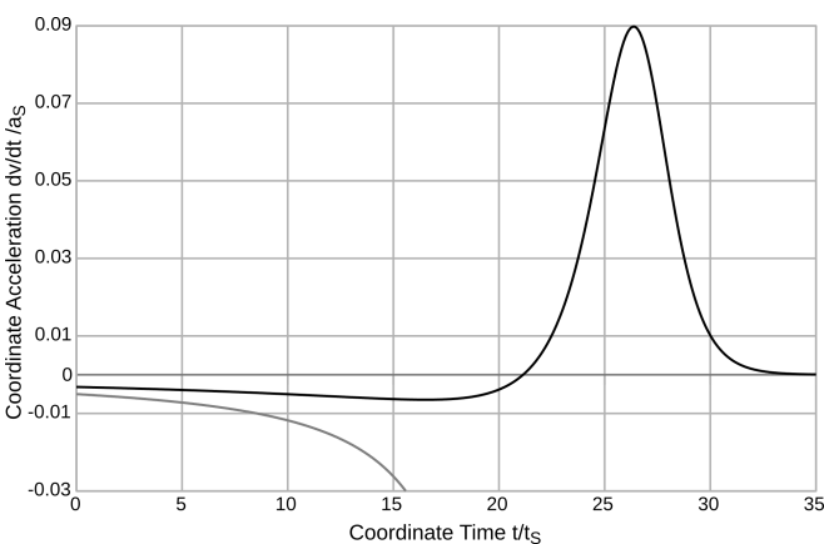




\section{DEVIATION MEASUREMENT}

The difference is difficult to measure experimentally. We can have an accelerated rocket, but its width $d$ is very small in front of the horizon distance $d_{H}$, and, consequently, the observed deviation is tiny. The deviation depends on the ratio $D=d / d_{H}$ and we perform a series expansions:

$$
X=R_{X} \sqrt{1-\left(D-Y_{C}\right)^{2} / R_{Y}^{2}}-1 \simeq \underbrace{\frac{D}{\tan \theta}}_{\text {rect.traj. }}-\underbrace{\frac{D^{2}}{2 \beta_{0}^{2} \sin ^{2} \theta}}_{\text {parabol.corr. }}+\underbrace{\frac{D^{3}}{2 \beta_{0}^{2} \sin ^{2} \theta \tan \theta}-\left[\frac{D^{4}}{8 \beta_{0}^{4}}\right]_{\theta= \pm \frac{\pi}{2}}+\ldots}_{\text {ellipticalcorrections }}
$$

The term in $D^{2}$ corresponds to the non-zero first order of the deviation due to the non-inertial character of the reference frame. The terms $D^{3}$ and beyond correspond to the deviation between classical theory and special relativity. For $d=10 \mathrm{~m}, a=10 \mathrm{~m} / \mathrm{s}^{2}$ and $\theta=45^{\circ}$, we have $|\Delta x| \simeq 11 \mathrm{fm}$ for the parabolic correction of a light ray, or, for the elliptic correction of a non-zero mass particle of velocity $v_{0}=10 \mathrm{~m} / \mathrm{s}$ (bell shot). The deviation is extremely small and a direct measurement seems out of reach.

On the other hand, with the precision of atomic clocks, the time deviation is experimentally accessible. For example, let's launch a clock upwards with a vertical motion. Let us consider a maximum height reached of $h=45 \mathrm{~m}$. On its return, when the moving clock falls back in free fall to the level of the clock that remained at X=0, with $a=10 \mathrm{~m} / \mathrm{s}^{2}$ and $v_{0} \simeq 30 \mathrm{~m} / \mathrm{s}$, the time difference between the two clocks is $\Delta t \simeq 10 \mathrm{f}$ f for a time of flight of $6 \mathrm{~s}$ : for $\theta=0$ an expansion with respect to $\beta_{0}$ gives $\Delta t / t_{H}=\tau / t_{H}-T \simeq \beta_{0}^{3} / 3$ with $H \simeq \beta_{0}^{2} / 2$, and $\Delta t \simeq v_{0}^{3} /\left(3 a c^{2}\right)^{[7]}$.

Fig. 16 Minkowski diagram $(T, X)$ for a vertical launch. $\tau / t_{H}$ as a function of $X$ draws a circle arc.

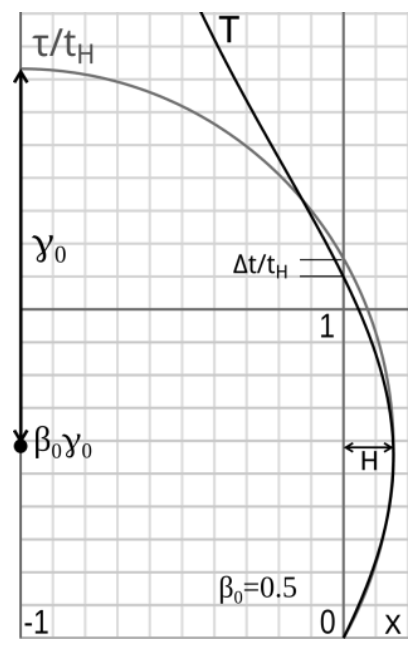

\section{CONCLUSION}

The calculations remain relatively simple and allow students to become familiar with concepts that are partly reused in general relativity. At the same time, this textbook case helps to avoid a lot of confusion in relativity. Pushing special relativity to its last non-inertial limits, where an underlying global Minkowskian framework persists, makes it easy to implement a progressive learning pedagogy. Moreover, the theoretical vision is broadened, and perhaps one day direct experimental verifications will be feasible.

[1] A. Einstein, Relativity, in Chapter A few inferences from the general theory of relativity, 1916.

[2] C. Møller, The Theory of Relativity, Oxford, 1st ed. 1952, See on page 253.

[3] E.A. Desloge, R.J. Philpott, Uniformly accelerated reference frames in special relativity, Am. J. Phys. 55, $252,1987$.

[4] E.A. Desloge, Spatial geometry in a uniformly accelerating reference frame, Am. J. Phys. 57, 598, 1989.

[5] W. Rindler, Relativity, Oxford University Press, 2nd ed. 2006, See on page 71.

[6] C. Semay, Observer with a constant proper acceleration, European Journal of Physics, 27(5), 1157-1167, 2006.

[7] M. Rouaud, Special Relativity, A Geometric Approach, voyagepourproxima.fr/SR.pdf, 2020, See p. 243 , 167 \& 387.

[8] J. Dwayne Hamilton, The uniformly accelerated reference frame, Am. J. Phys. 46, 83, 1978.

[9] A. Spallicci, P. Ritter, A fully relativistic radial fall, Int. J. of Geom. Meth. in Mod. Phys. 11(10), July 2014.

[10] E.A. Desloge, Nonequivalence of a uniformly accelerating reference frame and a frame at rest in a uniform gravitational field, Am. J. Phys. 57, 1121, 1989.

[11] S.I. Blinnikov, L.B. Okun, M.I. Vysotsky, Critical velocities $c / \sqrt{ } 3$ and $c / \sqrt{ } 2$ in the general theory of relativity, Physics-Uspekhi, 46(10), 1099-1103, October 2003.

[12] C.W. Misner, K.S. Thorne, J.A. Wheeler, Gravitation, W.H. Freeman \& Co., 1971, See on page 848.

[13] P. Tourrenc, Relativity and Gravitation, 1992, See on page 185. 